\title{
Confiabilidad y validez de constructo de la Escala de Modificación Corporal en varones adolescentes
}

\author{
Brenda Sarahi Cervantes-Luna, ${ }^{1}$ Georgina Alvarez-Rayón, ${ }^{2}$ Gilda Libia Gómez-Peresmitré, ${ }^{3}$ \\ Juan Manuel Mancilla-Díaz
}

Artículo original

\section{SUMMARY}

Several countries have noticed not only the adoption of body change strategies by males, but these may respond to different purposes: weight loss, weight gain and increased muscle tone or muscle mass. Nevertheless, instruments that allow the assessment of these aspects in a differentially way are scanty. Therefore, the aim of the present study was to obtain the Spanish version of the Body Modification Scale (BMS), as well as to examine its psychometric properties in adolescent men. The first study included the translation, adjustment and piloting of the BMS; internal consistency and factor structure were evaluated with 270 adolescents $\left(M_{\text {age }}=12.84\right) ; 171$ of them participated in retest. The second study was conducted to prove, in an independent sample $\left(n=200 ; M_{\text {age }}=13.46\right)$, the adequacy of the factor structure derived from the original. The Spanish version of the BMS indicated to have adequate internal consistency $(\alpha=.88)$ and test-retest reliability $(r=.80)$. The exploratory factor analysis derived initially four factors, showing conceptual overlap between two of them; therefore, it was opted to replicate the analysis performing an extraction to three factors. These explained $44.1 \%$ of the variance, grouping 20 of the 24 original items. The loss weight and increased muscle tone or muscle mass factors showed adequate reliability $(\alpha>86 ;>>80)$, resulting substantially lower the weight gain factor (.63 and .74 , respectively). The confirmatory factor analysis supported the adequacy of both structures; however, the model here proposed showed a better fit. In general, the Spanish version of the BMS showed adequate psychometric properties in adolescent men. Nevertheless, this should be corroborated not only in other ages, but also in women.

Key words: Body image, body weight change, reliability, validity, adolescents, men.

\section{RESUMEN}

En diversos países se ha constatado no sólo la adopción de estrategias de cambio corporal por parte de los varones, sino que éstas pueden responder a distintas intenciones: perder peso, ganar peso e incrementar tono o masa muscular. Sin embargo, son escasos los instrumentos que permiten evaluar diferencialmente estos aspectos. Por tanto, el propósito de esta investigación fue obtener una versión en español de la Body Modification Scale (BMS), así como examinar sus propiedades psicométricas en varones adolescentes. El primer estudio comprendió la traducción, adaptación y aplicación piloto de la BMS; la consistencia interna y estructura factorial fue evaluada con 270 adolescentes $\left(M_{\text {edad }}=12.84\right)$, de los cuales 171 participaron en el retest. El segundo estudio se dirigió a probar, en una muestra independiente $\left(n=200 ; M_{\text {edad }}=13.46\right)$, la adecuación de la estructura factorial derivada respecto a la original. La versión en español de la BMS mostró tener adecuada consistencia interna $(\alpha=.88)$ y confiabilidad test-retest $(r=80)$. El análisis factorial exploratorio derivó inicialmente cuatro factores, observándose traslapamiento conceptual entre dos de ellos; por tanto, se optó por replicar el análisis forzando la extracción a tres factores. Éstos explicaron $44.1 \%$ de la varianza, agrupando 20 de los 24 reactivos originales. Los factores perder peso y aumentar tono - masa muscular mostraron adecuada confiabilidad $(\alpha \geq .86 ; r \geq .80)$, resultando sustancialmente menor la del factor ganar peso (.63 y .74 , respectivamente). Los análisis factoriales confirmatorios fundamentaron la adecuación de ambas estructuras; sin embargo, el modelo correspondiente a la aquí propuesta mostró un mejor ajuste. En general, la versión en español de la BMS mostró poseer adecuadas propiedades psicométricas en varones adolescentes. Sin embargo, esto deberá corroborarse no sólo en otras edades, sino también en mujeres.

Palabras clave: Imagen corporal, cambio del peso corporal, confiabilidad, validez, adolescentes, varones.

FES-Iztacala, Universidad Nacional Autónoma de México; Centro Universitario-Ecatepec, Universidad Autónoma del Estado de México.

2 FES-Iztacala, Universidad Nacional Autónoma de México.

Facultad de Psicología, Universidad Nacional Autónoma de México.

Correspondencia: Dra. Georgina Alvarez Rayón. UNAM-FES Iztacala, División de Investigación y Posgrado (UIICSE). Av. de Los Barrios No. 1, Los Reyes Iztacala, 54090 Tlalnepantla, Estado de México, México. E-mail: alvarezr@unam.mx

Recibido primera versión: 2 de agosto de 2012. Segunda versión: 25 de julio de 2013. Aceptado: 11 de octubre 2013. 


\section{INTRODUCCIÓN}

La insatisfacción corporal ha sido descrita como uno de los factores clave para el desarrollo, mantenimiento y caracterización sintomática de ciertos trastornos mentales. Ejemplo de ello son los trastornos del comportamiento alimentario (TCA), caracterizados por el deseo excesivo de adelgazar y el miedo mórbido a la obesidad. ${ }^{1}$ Adicionalmente, en la década de 1990 se alertó sobre la existencia de otro síndrome al que ha dado en denominarse dismorfia muscular (DM), caracterizado por el deseo mórbido de incrementar la masa muscular., ${ }^{2,3}$

Si bien la definición de la DM en tanto entidad independiente o como una variante del trastorno dismórfico corporal o de los TCA es aún una asignatura pendiente, ${ }^{4,5}$ se han descrito estrechos paralelismos de estos últimos, específicamente la anorexia nerviosa y la bulimia nerviosa, con la $\mathrm{DM},{ }^{6,7}$ aunque también se les ha diferenciado a partir de las estrategias de cambio corporal asociadas a ellas, 8,9 prodelgadez o promusculatura, respectivamente.

En este contexto, las estrategias de modificación corporal son conductas que tienen como finalidad cambiar la forma, el tamaño o el peso corporal, ${ }^{10}$ y son adoptadas tanto por mujeres como por varones. ${ }^{11,12}$ Sin embargo, principalmente en estos últimos, dichas estrategias no sólo responden al deseo de adelgazar, sino además al de incrementar su masa o tono muscular, ${ }^{13-19}$ lo que responde a un ideal corporal basado en la posesión de fuerza y masa muscular. ${ }^{20,21}$

Con base en el ideal muscular, las conductas de modificación corporal pueden implicar desde modificaciones en la dieta, la práctica de ejercicio excesivo y el uso de sustancias $^{10,18,22,23}$ hasta el empleo de procedimientos quirúrgicos (e.g., colocación de implantes). ${ }^{24}$ Estas estrategias pueden desencadenar complicaciones no sólo médicas, sino también en la salud mental. ${ }^{25}$

Dadas las divergencias documentadas entre hombres y mujeres en cuanto a sus ideales corporales, ${ }^{26,27}$ interiorizados aun desde edades tempranas, ${ }^{28,29}$ surge la necesidad de contar con instrumentos válidos y confiables que permitan evaluar aquellas conductas de cambio corporal que no estén unívocamente dirigidas al adelgazamiento, sino también al deseo de incrementar peso corporal, o bien, específicamente al de aumentar masa o tono muscular. Al respecto, una revisión sistemática reciente advirtió que aún son incipientes los estudios que informan sobre el uso de medidas que permitan evaluar ambas vertientes del cambio corporal: proadelgazamiento y promusculatura. ${ }^{30}$ No obstante, fue posible identificar la existencia de un cuestionario de autorreporte específicamente desarrollado para tal fin: la Body Modification Scale (BMS). ${ }^{18}$

En varones adolescentes de entre 11 y 17 años de edad, McCabe y Vincent ${ }^{18}$ encontraron que la BMS posee excelente consistencia interna $(\alpha=.93)$, confirmando además, con base en el Análisis Factorial Exploratorio (AFE), la estructura prevista de tres factores: pérdida de peso $(\alpha=.99)$, incremento de peso $(\alpha=.98)$ y aumento de tono o masa muscular $(\alpha=.95)$, con ocho reactivos cada uno. Asimismo, la escala mostró tener adecuada estabilidad sobre el tiempo (test-retest), tanto en su puntuación total $(r=.90)$ como en sus factores: $.88, .92 \mathrm{y}$ .89 , respectivamente. Con base en ello, estas autoras sugieren que la BMS es una medida válida y confiable, aunque enfatizan la necesidad de que esto pueda corroborarse en futuros estudios. Por tanto, el propósito de la presente investigación fue obtener una versión en español de la Body Modification Scale, así como evaluar su confiabilidad y validez de constructo en varones adolescentes. Este trabajo consta de dos estudios. El primero está dirigido a explorar la consistencia interna, estabilidad en el tiempo y estructura factorial de la escala, mientras que el segundo se enfocó en comprobar la validez de dicha estructura. La presente investigación fue aprobada por un comité universitario de ética.

\section{ESTUDIO 1}

\section{Método}

Muestra

No probabilística de tipo intencional. El tamaño se calculó atendiendo la recomendación de contar con un número de observaciones 10 veces mayor que el de variables a analizar. ${ }^{31}$ Así, la muestra estuvo conformada por 270 varones adolescentes de entre 11 y 15 años de edad $(M=12.84$; $D E=0.99)$; todos residentes en la zona metropolitana de la Ciudad de México, y provenientes de dos centros escolares públicos. Mientras que en el retest participaron 171 de estos adolescentes $\left(M_{\text {edad }}=12.81\right.$ años; $\left.D E=1.03\right)$.

\section{Instrumento}

Escala de Modificación Corporal (BMS), ${ }^{18}$ la cual es un cuestionario de autorreporte constituido por 24 reactivos tipo Likert (1=nunca, $6=$ siempre), donde una puntuación más alta refleja mayor presencia del rasgo.

\section{Procedimiento}

1. Traducción. Se llevó a cabo la traducción de doble vía de la BMS (inglés-español, español-inglés), misma que fue revisada por tres expertos en el área, a fin de evitar una interpretación errónea de los reactivos. Finalmente, éstos fueron adaptados en cuanto a lenguaje, con el propósito de garantizar su comprensión por parte de los adolescentes.

2. Estudio piloto. Una vez que se obtuvo la versión final, se estableció contacto con las autoridades escolares, se les explicó el propósito de la investigación y se solicitó su autorización para llevar a cabo el levantamiento de datos. Posteriormente, a los alumnos se les solicitó participar en "una investigación sobre imagen corporal en adolescentes" y, a quienes accedieron voluntariamente, se les solicitó tanto su consentimiento informado como 
el de sus padres y/o tutores, garantizándoles el uso anónimo de los resultados.

Así, en el piloteo de la BMS participaron dos grupos de 15 adolescentes cada uno, con un rango de 11 a 14 años de edad $(M=12.00 ; D E=0.64)$. Durante la sesión de aplicación, las instrucciones y cada uno de los reactivos fueron leídos en voz alta al grupo, exhortando a los participantes a indicar si eran confusos o poco claros, o bien, si desconocían el significado de alguna de las palabras empleadas. Al respecto, los participantes refirieron que los reactivos eran claros y que no tenían problema para comprender el lenguaje empleado. Por tanto, no fue necesario realizar cambios al instrumento.

3. Recolección de datos. La aplicación de la BMS a la muestra principal se realizó dentro del horario escolar y de manera grupal (de entre 15 y 20 participantes), en una sesión con duración aproximada de 20 minutos. Para el caso del retest, los participantes completaron la escala en una segunda ocasión, que fue un mes después de la primera aplicación.

4. Análisis de datos. Se realizó con el Paquete Estadístico para las Ciencias Sociales (SPSS, versión 17.0 para Windows) y consistió en calcular la consistencia interna (coeficiente alpha de Cronbach) y la estabilidad de la medida en el tiempo (coeficiente $r$ de Pearson entre test-retest). Finalmente, la validez de constructo se valo- ró con AFE, mediante el método de extracción de ejes principales y rotación Varimax.

\section{Resultados}

El valor del coeficiente alpha de Cronbach indicó que la BMS cuenta con buena consistencia interna $(\alpha=.88)$. El coeficiente $r$ de Pearson indicó que los 24 reactivos se correlacionaron positiva y significativamente con la puntuación total (21 con $p=.0001)$. Con lo que respecta a la magnitud de la correlación, mientras que la de cuatro reactivos $(1,4,16$ y 22) fue excesivamente débil $(r \leq .29)$, la de 14 fue desde moderada hasta fuerte $(r \geq .50)$. La correlación test-retest de la puntuación total fue $.80(p=.0001)$.

De manera preliminar a la realización del AFE, se examinó la adecuación de los datos. La correlación inter-reactivos arrojó que el mayor coeficiente fue .71, constatándose la no colinealidad entre las variables; el índice de Kaiser-Meyer-Olkin (KMO) fue .86, mayor al mínimo recomendado de $.70 ; 32$ en tanto que la prueba de esfericidad de Barlett resultó significativa $\left(x^{2}=2895.21, \mathrm{p} \leq .0001\right)$. El AFE derivó cuatro factores con valor propio mayor a uno, mismos que explicaron $49.96 \%$ de la varianza total y, con base en el criterio de carga factorial mínima de .40 , agruparon 21 reactivos, con lo que resultaron excluidos tres alusivos al incremento de peso: 1 , 13 y 22 (cuadro 1). No obstante, se encontró que tanto en el segundo como en el cuarto factor se agruparon reactivos di-

Cuadro 1. Cargas factoriales de los reactivos en el primer AFE

\begin{tabular}{|c|c|c|c|c|}
\hline \multirow{2}{*}{ Reactivos } & \multicolumn{4}{|c|}{ Factores } \\
\hline & 1 & 2 & 3 & 4 \\
\hline 1 ¿Comes alimentos que te ayudarán a aumentar de peso? & & & & \\
\hline ¿Te preocupa estar demasiado gordo? & .58 & & & \\
\hline ¿Haces ejercicio con pesas para desarrollar o fortalecer tus músculos? & & .63 & & \\
\hline ¿Te gustaría que lo que comes te ayude a aumentar de peso? & & & .44 & \\
\hline ¿Haces ejercicio para bajar de peso? & .45 & .55 & & \\
\hline ¿Haces ejercicio para aumentar el tamaño de tus músculos? & & .77 & & \\
\hline ¿A propósito comes de más para aumentar de peso? & & & .64 & \\
\hline ¿Piensas en comer menos para bajar de peso? & .72 & & & \\
\hline ¿Piensas en tener músculos más grandes? & & .43 & & .59 \\
\hline ¿Te molesta que lo que comes no resulte en un incremento de peso? & & & .48 & \\
\hline 11 ¿Piensas en hacer ejercicio para bajar de peso? & .74 & & & \\
\hline 12 ¿Serías más feliz si tuvieras un cuerpo voluminoso, con músculos grandes? & & & & .69 \\
\hline 13 ¿ेHaces ejercicio para aumentar de peso? & & & & \\
\hline 14 ¿́la idea de bajar de peso siempre está en tu mente? & .83 & & & \\
\hline ¿Vas al gimnasio para aumentar el tamaño de tus músculos? & & .55 & & \\
\hline ¿Piensas en comer más para aumentar de peso? & & & .74 & \\
\hline ¿Comes alimentos bajos en grasas porque no quieres subir de peso? & .57 & & & \\
\hline ¿Piensas en hacer ejercicio para aumentar el tamaño de tus músculos? & & .53 & & .57 \\
\hline ¿A propósito comes entre comidas para subir de peso? & & & .59 & \\
\hline 20 ¿Cuidas lo que comes porque quieres estar más delgado? & .75 & & & \\
\hline 21 ¿Realizas ejercicio o algún deporte para aumentar el tamaño de tus músculos? & & .65 & & \\
\hline 22 ¿Piensas en hacer ejercicio para aumentar de peso? & & & & \\
\hline 23 ¿A propósito comes menos para bajar de peso? & .80 & & & \\
\hline $24 \dot{2}$ Te preocupa que tu cuerpo no sea lo suficientemente musculoso? & & & & .56 \\
\hline Valor propio & 4.27 & 2.75 & 2.04 & 1.89 \\
\hline Porcentaje de varianza explicada & 17.79 & 11.47 & 8.50 & 7.90 \\
\hline
\end{tabular}


rigidos a evaluar pensamientos y conductas vinculadas con el deseo de incrementar el tono o la masa muscular. Con base en estos resultados, y en que originalmente la BMS fue construida para medir las conductas de cambio corporal resultantes de tres distintas motivaciones (perder peso, ganar peso y aumentar tono o masa muscular), se decidió realizar un segundo AFE, forzando la extracción a tres factores.

Estos factores explicaron $44.06 \%$ de la varianza total y agruparon los mismos 21 reactivos (cuadro 2). No obstante, se observa que el reactivo 5 (referente a pérdida de peso) saturó en dos factores, con apenas una diferencia de .10 entre cargas factoriales, por lo que se decidió omitirlo. Así, con base en 20 de los 24 reactivos originales quedó definida la estructura tri-factorial de la BMS, quedando de la siguiente forma: perder peso, con siete reactivos $(2,8,11,14,17,20 \mathrm{y}$ $23)$; aumentar tono o masa muscular, con ocho reactivos $(3,6$, $9,12,15,18,21$ y 24); e incrementar peso, con cinco reactivos $(4,7,10,16$ y 19). La consistencia interna de estos factores fue satisfactoria, con los coeficientes alpha siguientes: $.89, .86$ y .74, respectivamente. La correlación entre los tres factores fue significativa entre los dos primeros factores $(r=.43, p=.0001)$, así como entre el segundo y tercero $(r=.27, p=.0001)$; pero no así entre este último y el primer factor $(r=.08, p=.21)$. Finalmente, se analizó la estabilidad test-restest de la puntuación en los factores $(n=171)$, obteniéndose los valores de $r$ siguientes: $.81, .80$ y .63 , respectivamente (todos con $p=.0001$ ).

\section{ESTUDIO 2}

\section{Método}

\section{Muestra}

No probabilística de tipo intencional. Participaron 200 varones adolescentes, con un rango de 12 a 16 años de edad $(M=13.46$; $D E=1.09)$. Todos residentes en la zona metropolitana de la Ciudad de México y provenientes de un centro escolar público.

\section{Instrumento}

Versión al español de la BMS derivada en el estudio previo.

\section{Procedimiento}

La aplicación de la escala se realizó de la forma ya descrita en el caso de la muestra principal del estudio 1.

1. Análisis de datos. Inicialmente se evaluó la consistencia interna (alpha de Cronbach); posteriormente se realizaron Análisis Factoriales Confirmatorios (AFC) mediante el Programa de Ecuaciones Estructurales (EQS, versión 6.1 para Windows). Éstos se efectuaron con base en el método de máxima verosimilitud y considerando los índices de optimización de Lagrange y Wald. El ajuste del modelo se valoró con base en los indicadores siguientes: $\mathrm{Chi}^{2}$, Chi ${ }^{2}$ normada (CN, con la fórmula: $\left.x^{2} / \mathrm{gl}\right)$, índice de ajuste no normado (NNFI), índice de bondad

Cuadro 2. Cargas factoriales de los reactivos en el segundo AFE

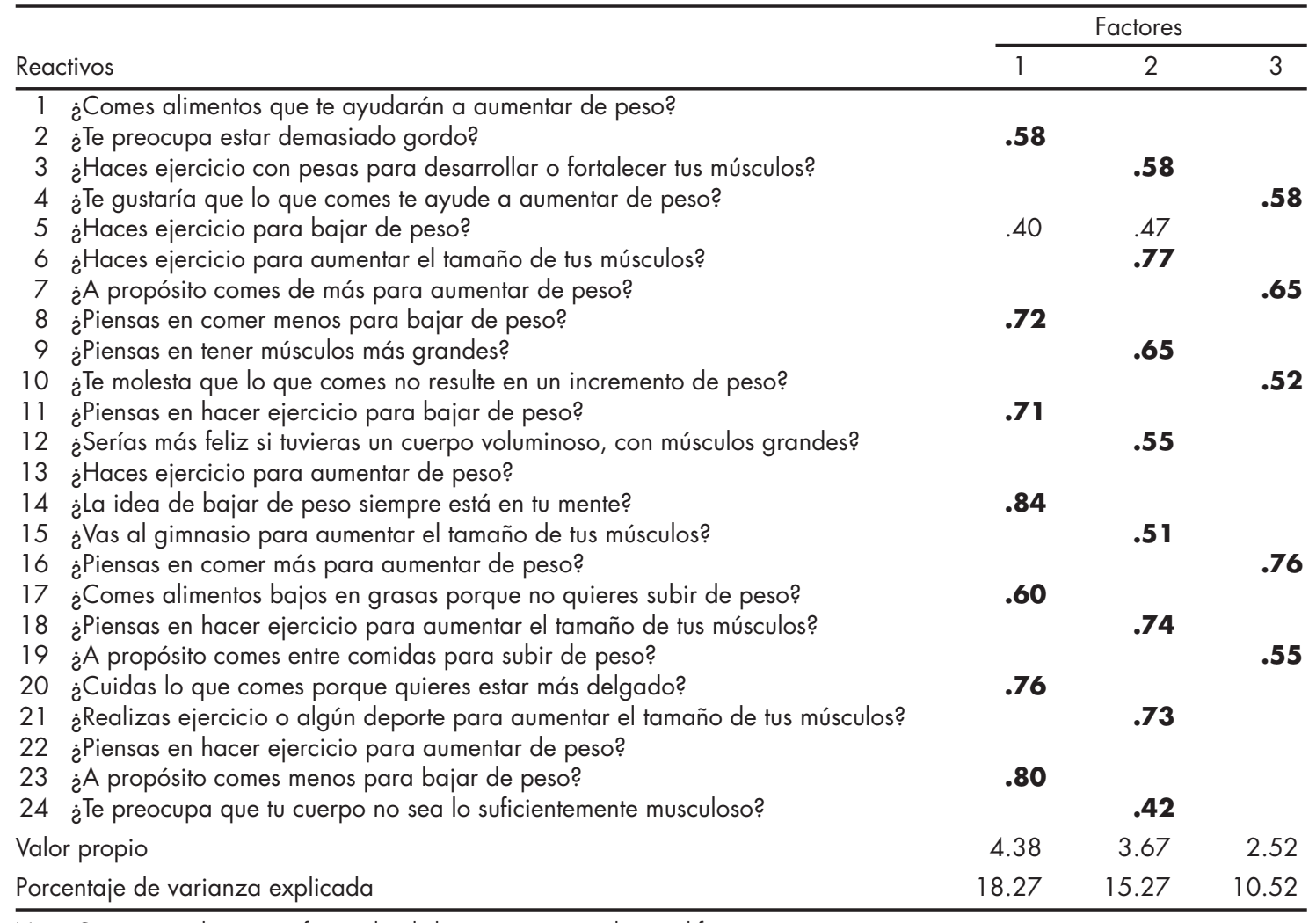

Nota. Con negritas las cargas factoriales de los reactivos retenidos en el factor. 
de ajuste (GFI), índice comparativo de ajuste de Bentler (CFI), raíz residual estandarizada cuadrática media (SRMR) y residuo cuadrático medio de la aproximación (RMSEA); considerando los criterios siguientes: $\mathrm{CN}<$ 3; ${ }^{33}$ NNFI, GFI y CFI $\geq .90 ;{ }^{34}$ SRMR $\leq .08$ y RMSEA $\leq .06 .{ }^{35,36}$

\section{Resultados}

La BMS mostró buena consistencia interna, tanto en el caso de su puntuación total $(\alpha=.88)$, como de sus tres factores ( $\alpha=.88, \alpha=.87$ y $\alpha=.72$, respectivamente). Respecto a la comprobación de la estructura factorial derivada en el estudio previo, el AFC arrojó un modelo que si bien tuvo una $\mathrm{Chi}^{2}$ significativa $\left(X^{2}, 153=229.68, p=.0002\right)$, los valores de $C N$ (1.50), NNFI (.95), GFI (.96), CFI (.90), SRMR (.08) y RMSEA (.05) indicaron un ajuste adecuado. Mientras que al probar en esta misma muestra la estructura de la BMS propuesta por McCabe y Vicent, ${ }^{18}$ el AFC derivó un modelo con menor bondad de ajuste $\left(X^{2}, 153=381.01, p<.0001 ; C N=2.49\right.$, $N N F I=.92, G F I=.93, C F I=.86, S R M R=.08, R M S E A=.06)$.

\section{DISCUSIÓN}

De acuerdo con los resultados del presente estudio, la versión al español de la BMS demostró ser un instrumento confiable para evaluar las estrategias de modificación corporal en varones adolescentes. No obstante, es importante puntualizar que si bien la escala mostró poseer adecuada consistencia interna y estabilidad sobre el tiempo, ambos coeficientes fueron sustancialmente menores a los reportados por McCabe y Vincent. ${ }^{18}$

Con lo que respecta a la estructura factorial de la escala, se confirma que está conformada por los tres factores propuestos: ${ }^{18}$ perder peso, ganar peso e incrementar tono o masa muscular. Sin embargo, en el presente estudio se observaron ciertas divergencias con respecto a la estructura original, que fueron: 1 . Los factores sólo agruparon 20 de los 24 reactivos; 2 aunque explicaron un porcentaje de la varianza superior a $40 \%$, fue sustancialmente menor al previamente reportado; ${ }^{18} 3$. no obstante, los valores propios y las varianzas explicadas por los factores derivados mostraron un sustancial mayor balance que en el estudio original, ${ }^{18}$ donde sólo el primer factor explicó $37.7 \%$ de la varianza total; y 4. cambió el orden de los factores, de modo que perder peso mostró ser el más relevante, seguido del referente a ganar tono o masa muscular y, por último, el de ganar peso.

Específicamente, tres de los cuatro reactivos no agrupados -dado que no cumplieron con el criterio de carga factorial mínima- estaban dirigidos a aumentar de peso, dos de ellos por medio de la realización de ejercicio. Al respecto, pareciera claro que los varones adolescentes pueden identificar dos finalidades de la realización de ejercicio como estrategia de cambio corporal: para adelgazar o para incre- mentar tono/masa muscular, pero no para aumentar peso corporal. Contrariamente, un reactivo referente a la pérdida de peso a través de la realización de ejercicio fue descartado en razón de que presentó carga factorial similar en dos factores: perder peso e incrementar tono o masa muscular. Esto puede ser resultado de que en este estudio, como en otros previos, se encontró que ambas intencionalidades están significativamente correlacionadas, ${ }^{8,14,16,37-39}$ coexisten,, 11 e incluso se ha encontrado que las primeras pueden predecir a las segundas, ${ }^{40}$ una vez que en sí mismo el ideal corporal masculino supone tanto la reducción de la grasa corporal como el incremento del tono y/o masa muscular.

En este estudio no se encontró que los factores ganar peso y perder peso estuvieran significativamente asociados, como se ha reportado en otros estudios también realizados con varones. ${ }^{18,38}$ Además, contrariamente a lo reportado por McCabe y Vincent, ${ }^{18}$ sólo los factores perder peso y aumentar tono o masa muscular de la BMS mostraron adecuada consistencia interna y estabilidad en el tiempo; en tanto que en el caso del factor ganar peso, incluyendo únicamente preguntas relativas a la ingestión de alimento, ambas propiedades fueron apenas satisfactorias.

Con lo que respecta a los AFC realizados, el modelo con los tres factores de la BMS aquí derivados mostró un mejor ajuste que el logrado con la conformación original de los factores o que el reportado por McCabe y Vincent. ${ }^{18} \mathrm{Si}$ bien en estos tres modelos el estadístico Chi-cuadrada resultó significativo, el correspondiente a la conformación de factores propuesta en el presente estudio mostró mejores índices de ajuste, particularmente al compararlos respecto a los obtenidos por dichas autoras y, más específicamente, en relación con el índice Chicuadrada normada, cuyo valor (6.3) fue excesivamente mayor al máximo sugerido. ${ }^{33}$ Por tanto, si bien en lo general se confirma la validez de la estructura trifactorial de la BMS en varones adolescentes, en el presente estudio se fundamenta una reestructuración en los reactivos contenidos en sus factores.

Finalmente, es importante señalar que estos resultados sólo suponen una primera evaluación de las propiedades psicométricas de la versión en español de la BMS, por lo que será importante que futuros estudios puedan realizar la comprobación de la estructura propuesta en una muestra de mayor tamaño, así como fundamentar su utilidad en la evaluación de las conductas de cambio corporal no sólo en varones de otras edades, sino también entre poblaciones del sexo femenino. Asimismo, es necesario puntualizar que si bien la BMS parece ser una herramienta apta para la evaluación de las conductas dirigidas al cambio corporal (en términos de peso o forma), no tiene como propósito identificar los síntomas de los trastornos de la conducta alimentaria o de la dismorfia corporal y, menos aún, diagnosticarlos. Además, al respecto, habrá que recordar que si bien los instrumentos de autorreporte son de gran utilidad en el campo de la salud mental, la formulación de un diagnóstico sólo puede derivarse de la entrevista conducida por un especialista. 


\section{AGRADECIMIENTOS}

Esta investigación forma parte de la tesis doctoral de la primera autora, quien agradece al CONACyT la concesión de la beca 33902; además fue financiada con los fondos otorgados por UNAM-DGAPA-PAPIIT (IN305912) y CONACyT (131865-H).

\section{REFERENCIAS}

1. Mancilla JM, Gómez-Peresmitré G, Alvarez G, Franco K et al. Trastornos del comportamiento alimentario en México. En: Mancilla JM, Gómez-Peresmitré G (eds). Trastornos alimentarios en Hispanoamérica. México: Manual Moderno y Universidad Nacional Autónoma de México; 2006.

2. Pope HG, Gruber AJ, Choi P, Olivardia R et al. Muscle dysmorphia: An underrecognized form of body dysmorphic disorder. Psychosomatics 1997;38:548-557.

3. Pope HG, Katz DL, Hudson JL. Anorexia nervosa and "reverse anorexia" among 108 male bodybuilders. Compr Psychiatry 1993;34(6):406-409.

4. Murray SB, Rieger E, Touyz SW, De la Garza Y. Muscle Dysmorphia and the DSM-V conundrum: Where does it belong? A review paper. Int J Eat Disord 2010;43:483-491.

5. Nieuwoudt JE, Zhou S, Coutts RA, Booker R. Muscle dysmorphia: Current research and potential classification as a disorder. Psychol Sport Exerc 2012;13:69-577.

6. Alvarez G, Escoto MC, Vázquez R, Cerero LA et al. Trastornos del comportamiento alimentario en varones: De la anorexia nerviosa a la dismorfia muscular. En: López A, Franco K (eds). Comportamiento alimentario: Una perspectiva multidisciplinar. México: Universidad de Guadalajara; 2009.

7. Murray SB, Rieger E, Hildebrandt T, Karlov L et al. A comparison of eating, exercise, shape, and weight related symptomatology in males with muscle dysmorphia and anorexia nervosa. Body Image 2012;9:193-200.

8. Bratland-Sanda S, Sundgot-Borgen J. symptoms of eating disorders, drive for muscularity and physical activity among norwegian adolescents. Eur Eat Disorders Rev 2012;20:287-293.

9. Kelley CC, Neufeld JM, Musher-Eizenman DR. Drive for thinness and drive for muscularity: Opposite ends of the continuum or separate constructs? Body Image 2010;7:74-77.

10. McCabe MP, Ricciardelli LA. Body image and body change techniques among youth adolescent boys. Eur Eat Disord Rev 2001;9:335-347.

11. Bottamini G, Ste-Marie DM. Male voices on body image. Inter J Men's Health 2006;5(2):109-132.

12. Ricciardelli LA, McCabe MP. Children's body image concerns and eating disturbance: A review of the literature. Clin Psychol Rev 2001;21:325-344.

13. Brunet J, Sabiston CM, Dorsch KD, McCreary DR. Exploring a model linking social physique anxiety, drive for muscularity, drive for thinness and self-esteem among adolescent boys and girls. Body Image 2010;7:137-142.

14. Carlson D, Bain N, King S. Weight and muscularity concerns as longitudinal predictors of body image among early adolescent boys: A test of the dual pathways model. Body Image 2008;5:195-204.

15. Kostanski M, Fisher A, Gullone E. Current conceptualization of body image dissatisfaction: Have we got it wrong? J Child Psychol Psychiatry 2004;45(7):1317-1325.

16. McCabe MP, Ricciardelli LA. A longitudinal study of body change strategies among adolescent males. J Youth Adolesc 2003;32(2):105-113.

17. McCabe MP, Ricciardelli LA, Finemore J. The role of puberty, media and popularity with peers on strategies to increase weight, decrease weight and increase muscle tone among adolescent boys and girls. J Psychosom Res 2002;52:145-153.

18. McCabe MP, Vincent MA. Development of Body Modification and Excessive Exercise Scale for adolescents. Assessment 2002;9(2):131-141.

19. Muris P, Meesters C, Van der Blom W, Mayer B. Biological, psycholo- gical, and sociocultural correlates of body change strategies and eating problems in adolescent boys and girls. Eat Behav 2005;6(1):11-22.

20. Hargreaves DA, Tiggemann M. Body image is for girls: A qualitative study of boys' body image. J Health Psychol 2006;11:567-576.

21. Thianthai C. Do male and female adolescents view their dissatisfaction with body parts in the same way? Int J Adolesc Med Health 2008;20(1):33-39.

22. Field AE, Austin SB, Camargo CA, Taylor CB et al. Exposure to the mass media, body shape concerns, and use of supplements to improve weight and shape among male and female adolescents. Pediatrics 2005;116(2):214-220.

23. Smolak L, Levine MP. Body image in children. En: Thompson JK, Smolak L (eds). Body image, eating disorders, and obesity in youth: Assessment, prevention and treatment. Washington DC: American Psychological Association;2002.

24. Sarwer D, Crerand CE, Gibbons LM. Cosmetic procedures to enhance body shape and muscularity. En: Thompson JK, Cafri G (eds). The muscular ideal: Psychological, social and medical perspectives. Washington DC: American Psychological Association; 2007.

25. Knoesen N. To be Superman: The male looks obsession. Aust Fam Physician 2009;38(3):131-133.

26. Gómez-Peresmitré G, Granados A, Jáuregui J, Tafoya $S$ et al. Trastornos de la alimentación: Factores de riesgo en muestras del género masculino. Psicol Contemporánea 2000;7(1):4-15.

27. Mulasi-Pokhriyal U, Smith C. Assessing body image issues and body satisfaction/dissatisfaction among Hmong American children 9-18 years of age using mixed methodology. Body Image 2010;7:341-348.

28. Baghurst T, Carlson D, Wood J, Wyatt FB. Preadolescent male perceptions of action figure physiques. J Adolesc Health 2007;41:613-615.

29. Harrison K, Bond BJ. Gaming magazines and the drive for muscularity in preadolescent boys: A longitudinal examination. Body Image 2007;4:269-277.

30. Cervantes BS, Alvarez G, Mancilla JM, Escoto MC. Prevención de insatisfacción corporal y conductas anómalas de cambio corporal en varones: Una revisión sistemática. Rev Mex Psicol 2011;(número especial octubre):194-195.

31. Hair JF, Black WC, Babin BJ, Anderson RE. Multivariate data analysis. Séptima edición. New Jersy: Prentice Hall; 2009.

32. Visauta B, Martori J. Análisis estadístico con SPSS para Windows: Estadística multivariante. Vol. 2. Madrid: McGraw-Hill; 2003.

33. Carmines E, McIver J. Analyzing models with unobserved variables: Analysis of covariance structures. En: Bohrnstedt G, Orgatta E (eds). Social measurement: Current issues. Beverly Hills, CA: Sage; 1981.

34. Byrne BM. Structural equation modeling with Amos: Basic concepts, applications and programming. Mahwah, NJ: Erlbaum; 2001.

35. Hu L, Bentler PM. Cut-off criteria for fit indexes in covariance structure analysis: Conventional criteria versus new alternatives. Structural Equation Modeling 1999;6:1-55.

36. Steiger JH. Structural modeling evaluation and modification: An interval approach. Multivar Behav Res 1990;25:173-180.

37. McCabe MP, Ricciardelli LA, Banfield S. Body image, strategies to change muscles and weight, and puberty: Do they impact on positive and negative affect among adolescent boys and girls? Eat Behav 2001;2:129-149.

38. Ricciardelli LA, McCabe MP. Psychometric evaluation of the Body Change Inventory: An assessment instrument for adolescent boys and girls. Eat Behav 2002;3:45-59.

39. Tylka TL. Refinement of the tripartite influence model for men: Dual body image pathways to body change behaviors. Body Image 2011;8:199-207.

40. McCabe MP, Ricciardelli LA. A longitudinal study of pubertal timing and extreme body change behaviors among adolescent boys and girls. Adolescence 2004;39:145-166.

Artículo sin conflicto de intereses 